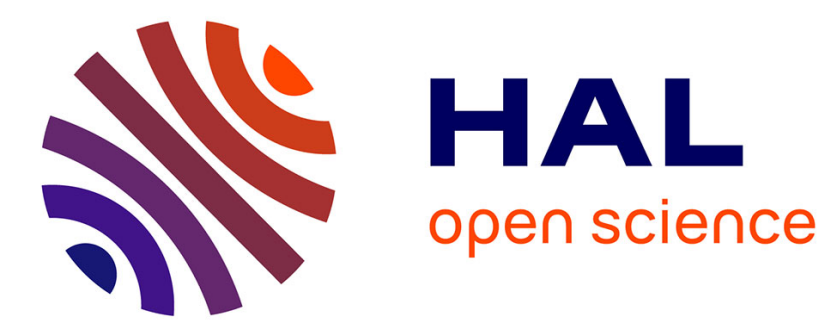

\title{
Dynamically Personalizing Search Results for Mobile Users
}

\author{
Ourdia Bouidghaghen, Lynda Tamine, Mohand Boughanem
}

\section{To cite this version:}

Ourdia Bouidghaghen, Lynda Tamine, Mohand Boughanem. Dynamically Personalizing Search Results for Mobile Users. Flexible Query Answering Systems-2009, Oct 2009, Roskild, Denmark. pp.99110, 10.1007/978-3-642-04957-6_9. hal-00476157

\section{HAL Id: hal-00476157 https://hal.science/hal-00476157}

Submitted on 23 Apr 2010

HAL is a multi-disciplinary open access archive for the deposit and dissemination of scientific research documents, whether they are published or not. The documents may come from teaching and research institutions in France or abroad, or from public or private research centers.
L'archive ouverte pluridisciplinaire HAL, est destinée au dépôt et à la diffusion de documents scientifiques de niveau recherche, publiés ou non, émanant des établissements d'enseignement et de recherche français ou étrangers, des laboratoires publics ou privés. 


\title{
Dynamically Personalizing Search Results for Mobile Users
}

Ourdia Bouidghaghen, Lynda Tamine-Lechani, and Mohand Boughanem

\author{
IRIT, CNRS - University of Toulouse \\ 118 route de Narbonne, \\ 31062 Toulouse Cedex 9, France \\ \{bouidgha, Lynda.Lechani, bougha\}@irit.fr
}

\begin{abstract}
We introduce a novel situation-aware approach to personalize search results for mobile users. By providing a mobile user with appropriate information that dynamically satisfies his interests according to his situation, we tackle the problem of information overload. To build situation-aware user profile we rely on evidence issued from retrieval situations. A retrieval situation refers to the spatio-temporal context of the user when submitting a query to the search engine. A situation is represented as a combination of geographical and temporal concepts inferred from concrete time and location information by some ontological knowledge. User's interests are inferred from past search activities related to the identified situations. They are represented using concepts issued from a thematic ontology. We also involve a method to maintain the user's interests over his ongoing search activity and to personalize the search results.
\end{abstract}

Keywords: Mobile information retrieval, mobile user, situation-aware profile, dynamic profile, time, location, personalization.

\section{Introduction}

The proliferation of mobile technologies such as (PDAs and mobile phones, ...) has made access to huge and heterogeneous collection of documents on the web, possible anywhere and anytime. This brings big challenges for researches in the information retrieval (IR) domain. Indeed, constraints and technical features of the mobile devices (difficulties of query input, limited display zone) yield to search practices which are different from that of the traditional desk queries. Studies on $\operatorname{logs}$ of mobile Internet user queries [1] show that user queries are shorter (thus more ambiguous), that there are fewer requests by session and fewer users who consult farther than the first page of the results list. Furthermore, according to studies in [2], $72 \%$ of the information needs of mobile users are related to contextual factors such as user interests, location and time.

Recent works in IR community attempt to improve search accuracy in this environment([3], 4] ). A first category of works has addressed issues related to the limited functionality of mobile devices, approaches have been proposed to facilitate query input $([5], 6])$, and to improve the search results visualization quality ([7, [8]).

T. Andreasen et al. (Eds.): FQAS 2009, LNAI 5822, pp. 99 110, 2009.

(C) Springer-Verlag Berlin Heidelberg 2009 
Another category of works aims at personalizing search results. Personalization aims to filter large amounts of information and returns a view on the information which matches the user's preferences and interests improving therefore the precision of the search results. Personalizing IR process in this environment is a key concern, this includes the need to provide information tailored to an individual user and taking into account contextual factors influencing his perception of what is relevant information. While some works use only the user feedback to build the user profile ([9], [10]), others ([11, [12]) use contextual information issued from his external environment, as an additional source of evidence to build some dynamic user profiles.

In order to endow personalized IR system with the capability to provide specifically a mobile user with information that match his interests coupled with his situation, we extend in this paper our previous work [13] on building and learning the user profile. We enhance in our approach the search context by spatiotemporal annotations, namely the location of the user and the time during his search activity. Our contribution consists in abstracting from sensor data some semantic information to characterize situations in which a user submits a query to the IR system. A user profile is learnt for each identified situation, on the basis of past search activities occurred in this situation. A method is also involved to dynamically select the most appropriate profile for personalizing search results by comparing the current situation with the past ones.

The paper is organized as follows. Section 2 reviews some related works. Section 3 presents our approach for representing and building a situation-aware user profile for a mobile user of search engines. Section 4 presents our method for exploiting the user profile in a personalized search. The last section presents our conclusion and points out possible direction for future work.

\section{Related Work}

A considerable amount of research has been done in providing personalized information for mobile users. Earlier personalization techniques (9], 10, 14]) are based solely on the computational behavior of the user (visited URL, viewed documents) to model his interests regardless of his surrounding environment (location, time, near people). The main limitation of such approaches is that they do not take into account the dynamicity of user interests regarding his situation. Indeed, mobile user interests particularly change according to his situation. To the best of our knowledge, few research works, summarized below, attempted to tackle this limitation.

In [1] the authors combine situation-based adaptation and profile-based personalization in the IR model. A situation is the result of a classification on a set of past contextual attributes (such as location, time, light, etc.) and/or actions (navigation, reading, etc). A user profile is generated for each situation and represented by the most frequent words extracted from documents related to the previous activities. Considering a user query, the related context is compared with the previous situations, once the situation identified, the query is refined with the corresponding profile. 
In [12] the authors propose a method for personalizing a portal for search services for mobile users. Their method consists of building a dynamic profile based on time and user experience, where the user preferences and interests are weighted according to temporal zones. The temporal zones are learnt by the study of the daily routine of the user and his activities for every period. To model the change of user's preferences according to his experiences (normal day, vacation), the weight association for the concepts in the user profile is established for every new experience of the user.

In [15] the authors propose a method for situational personalization, where a situation is represented by a combination of contextual data in particular the GSM cell identifier (CID) to which the user is connected, the MACs addresses of near Bluetooth devices (BT) and the time. Their experiments showed that it is possible to identify situations on the basis of the logs of the CID and the number of the near BT addresses. By using machine learning based methods, it is possible to derive from the CIDs and BTs, the situations on the basis of groups of BTs identified together in a rather frequent way for a given time period.

In [16] the authors address the idea of building a profile per region visited by a person in order to enhance targeting of advertisements to mobile users. They propose to combine primarily logs of cell towers visited with specific queries to the user (e.g., to ask what kinds of activities the user does in a given frequented region). They provide statistical techniques to determine information about visited regions, along with the frequency of visits, typical durations, and typical visit times. The profile content is built based on a user questionnaire.

In [17] the authors propose a context-aware and personalized mobile recommender system for young people in leisure time. The system predicts the user's current and future leisure activity (eating, seeing, reading, doing, and shopping) from context (time, location) and patterns of user behavior. The predicted user activity combined with models of the user's preferences, are used together to filter and recommend relevant content.

This paper presents a new approach for a situation-aware personalized search. In our approach we exploit both search history and diverse ontologies (location, time and thematic) to learn user's search situations and their corresponding user's interests. Comparatively to previous works, our approach has the following new features:

- A semantic representation of the user search situations as concepts from location and time ontologies with their corresponding user's interests as concepts from a global ontology, while in [11, 15] the user situation is represented by low level data and in [1] the user's interests are represented by simple key-words.

- Our approach is implicit and automatic; no effort is needed from the user, while in [12], 16] the user is solicited in the process of building his profile.

- Our approach does not take any restriction on user's situations or population, while in [17] it is devoted to some specific situations and specific populations. 


\section{Building a Situation-Aware User Profile}

\subsection{General Approach and Motivation}

While a user profile may contain different types of information [18, in our work, user profile refers to his domains of interests. Indeed, the user domains of interest are the most important contextual factor identified that alleviates an ambiguous web search in ad hoc retrieval task 19. In mobile IR, the computing environment is continuously changing due to the inherent mobility framework. More specifically, mobility induces user's information needs weakly dependent on his situation. In other words, the user's profile may change anytime due to change in user's environment (location, time, near person, etc). Just for example, assume that a person being at beach submits the query "sport"; knowing that he is interested both in skiing and surfing, we can improve search results by taking into account his interests for surfing and not for skiing given that he is at beach and not on a mountain. Static approaches for building the user profile are therefore poorly useful, so we rather focus on more dynamic techniques, any time capable of adjusting the user interests to the current search situation. In our work, a situation is a set of past (or current) attributes. More specifically, we consider two types of attributes time and location to distinguish mobile user situations when submitting a query to the search engine.

In this paper, we propose our situation-aware profile approach that can be used to adapt search results according to user's information needs in a certain situation. A user $U$ is represented by a set of situations with their corresponding user profiles, denoted : $U=\left\{\left(S^{i}, C^{i}\right)\right\}$, where $S^{i}$ is a situation and $C^{i}$ its corresponding user profile. Our work is driven by the assumption that in similar search situations, user's interests are likely to be similar. We detail in the following our methods for inferring user search situations from low level information, for building the user profiles, and for dynamically selecting the adequate user profile according to the current situation to be used to re-rank the search results.

\subsection{Situation Modeling}

Our challenge when building this situation-aware profile is to use sensory data to identify a user situation. We propose to associate low level information directly acquired from sensors to semantic concepts extracted from temporal and spatial ontologies. Hence, instead of knowing that a user is at location "48.7818034, 2.2183314" and time "Tue Aug 3 12:10:00 CEST 2008" we derive that he is "at beach, summer, holiday, midday". Our situation model is then represented by an aggregation of four dimensions :

- Location type: refers to class name (such as beach, school,... ) extracted from a classification category of location types (like ADL feature type thesauru: 1 ).

- Season: refers to one of the year's seasons.

- Day of the week: refers either to workday, weekend or holiday.

- Time of the day: refers to time zone of a day such as morning, night, ...

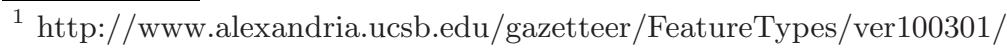


More specifically, a situation $S$ can be represented as a vector whose features $X$ are the values assigned to each dimension:

$$
S=\left(X_{l}, X_{u}, X_{v}, X_{w}\right)
$$

Where $X_{l}$ (resp. $X_{u}, X_{v}, X_{w}$ ) is the value of the location type (resp. season, day of the week and time of the day) dimension. Below, we give an outline of the location and time models on which the situation model relies.

Location Modeling. For mobile applications, the location aspect is of utmost importance. Location typically represents the point in space, where the user of the application is located. As discussed in 20, there are different plausible and correct ways to answer the question: where is the user located? and consequently different ways to characterize a location. As returned by location sensor systems (like GPS), location is an absolute position in some geographic coordinates systems. However, user behavior is unlikely to be conditioned by coordinates per se, but rather by what (else) is at these coordinates. Thus, we consider, in our work, a location class label (or named class) as relevant for our purpose of characterizing a situation of search. Such named classes are generally functional (like "yellow pages" naming), more importantly, a label directly represents the place's demographic (school), environmental (beach), historic (monument), personal (residence) or commercial (shop) significance and is the desired abstraction for our situation identification task. Simple automated place labeling is already commercialized (Google map, Yahoo local, Map-Point, ... ), it consists of merging web data such as postal addresses with maps witch enables Nearest-X services. Also, manual place classification is practiced in most geographic information systems like the Alexandria Digital Library 2 and GeoNames 3 servers. To insure the connection between the location coordinates and its semantic classification, a conceptual model is necessary to represent and reason about location. As in the SPIRIT 4 project, we use a spatial data base (as geo services) and a spatial thesaurus to represent and reasoning on geographic information. Fig. 1 shows a simplified model for representing spatial information.

The mapping between the concrete class "Footprint" (geographic coordinates) and the abstract class "Geographic Place" (generally expressed as "reverse geocoding operation") allows us to relate pure geographic coordinates to semantic places represented by their name and type (or class label). Also geographic places are related by different spatial relations such as contains, part-of, etc.

Time Modeling. The temporal information is complex; it is continuous and can be represented at different levels of granularity. To define the temporal aspects characterizing the situation a user is in, we suggest abstracting the continuum time into some specific and significant periods (abstract time classes), which we

\footnotetext{
${ }^{2}$ http://www.alexandria.ucsb.edu/

${ }^{3}$ http://www.geonames.org/

${ }^{4}$ Spatially-Aware Information Retrieval on the Internet:

http://www.geo-spirit.org/index.html
} 


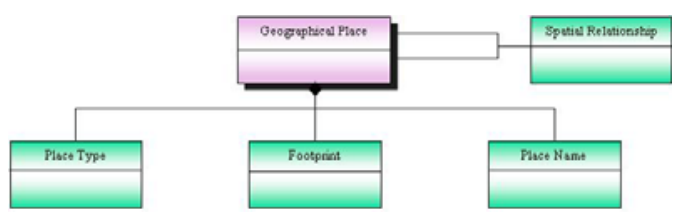

Fig. 1. A simple schema of the location model

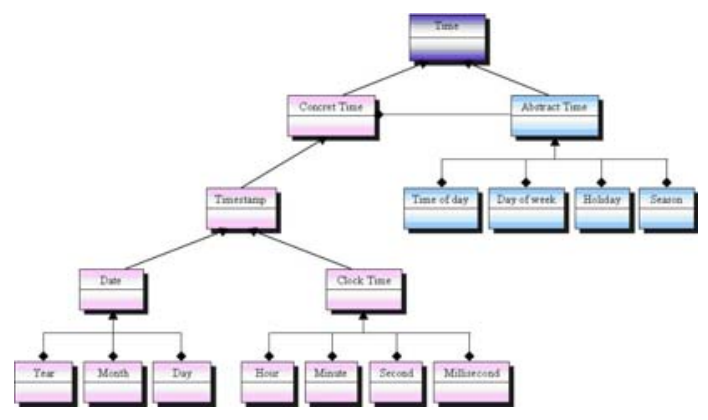

Fig. 2. A simple schema of the time model

expect having an effect on the user behavior (e.g. morning, weekend, winter). To allow a good representation of the temporal information and its manipulation, we propose to use OWL-Time ontology [21] and to extend it with some special classes of time: time of day, day of week and season(as shown in Fig. 2).

The time ontology abstracts a time point to a set of significant time intervals of our daily life, the mapping between the two is implemented by axioms and predicates. For the time of day class we define six periods: waking-time, morning, midday, afternoon, evening, night. For the day of week class we distinguish workdays (Mon, Tue, Thu, Wed, Fry), and rest-days composed of weekends (Sat, Sun) and holidays (any day on which work is suspended by law or custom).

\subsection{User Profile Modeling}

Below, we give an overview of the concept-based representation of the user profile developed in our previous work [13. A user profile is built at the end of each search activity. A search activity expresses the following events: the user submits a query to a search engine; the latter returns a ranked list of documents, then the user expresses his preferences on the document of his interests. We assume that a document retrieved by the search engine is relevant if it generates some observable user behaviors (page dwell time, click through, saving, etc).

Building a Keyword User Profile. Let $q^{s}$ be the query submitted by a specific user at time $s$. Let $D^{s}$ be the set of relevant documents returned with respect to the query $q^{s}$, which is represented as a single term vector using the $t f * i d f$ weighting scheme. The keyword user profile $K^{s}$ is a single term vector 
that represents the centroid of the documents in $D^{s}$, where the weight of a term $t$ is computed as follows:

$$
K^{s}(t)=\frac{1}{\left|D^{s}\right|} \sum_{d \in D^{s}} w_{t d} .
$$

where $w_{t d}$ is the weight of term $t$ in document $d$. In order to enhance the flat representation of the keyword user profile, a concept-based user profile is built by first mapping it on reference ontology, then disambiguating the mapped concepts set using a sub-concepts aggregation scheme.

Mapping the Keyword User Profile on the Ontology. The keyword user profile $K^{s}$ is mapped on the ODP5 ontology in order to extract a set of concepts that reflect semantically the user interest. Each concept of the ODP is related to sub-concepts with "is-a" relations and is associated to a set of web pages classified under that concept. Each concept is presented by a single term vector $\overrightarrow{c_{j}}$ extracted from all individual web pages classified under that concept as well as all of its sub concepts. Strategy involved briefly consists of creating a super-document $S d_{j}$ for each concept $c_{j}$ by concatenating the first 60 titles and descriptions associated to the web pages classified under that concept. Then stop words are removed and porter stemming algorithm is applied on the collection of super-documents. Finally, each concept $c_{j}$ is represented as a single term vector $\overrightarrow{c_{j}}$ where each term's weight $w_{i}$ is computed using $t f * i d f$ weighting scheme. Specifically, $t f$ is the total term frequency in the superdocument $S d_{j}$ as well as in the superdocuments associated to its sub-concepts. Given a concept $c_{j}$ of the ODP, represented by the term vector $\overrightarrow{c_{j}}$, its similarity weight $s w\left(c_{j}\right)$ with $\overrightarrow{K^{s}}$ is computed as follows:

$$
s w\left(c_{j}\right)=\cos \left(\overrightarrow{c_{j}}, \overrightarrow{K^{s}}\right) .
$$

Disambiguating the Mapped Concepts Set. Disambiguating the mapped concepts is carried out using a sub-concepts aggregation scheme, which relies on the assumption that the most relevant concepts are those having a greater number of descendant concepts mapped according to the ontology. We outline that the depth two of the ontology is too general to represent the user interests, and leaf nodes are too specific to improve retrieval precision for related search activities. So interest was ported on representing the user profile using depth three of the ODP ontology. A cluster of weighted concepts having a common general depth-three concept is first identified; its relevance score is computed by adding the weights of its descendant concepts. The weight of a general concept $c_{j}$, having a set of $n$ related descendant concepts $S\left(c_{j}\right)$, is computed as follows:

$$
s w\left(c_{j}\right)=\frac{1}{n} . \sum_{1 \leq k \leq n \wedge c_{k} \in S\left(c_{j}\right)} s w\left(c_{k}\right) .
$$

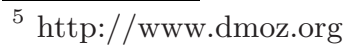


Finally, the user profile $C^{s}$ performed at time $s$ is represented as a set of depththree weighted concepts, noted $<c_{j}, p\left(c_{j}\right)>$.

\section{CBR Approach for Personalization}

While the same user $U$ can have many profiles, $U=\left\{\left(S^{i}, C^{i}\right)\right\}$, one of these profiles is the one primarily corresponding to the current user's activity and query. In order to select the most adequate user profile to be used for personalization, we compare the similarity between a new search situation and the past ones. Comparing past user experiences is referred to in the literature as case-based reasoning (CBR) 22]. In CBR a problem is solved based on similar solutions of past problems. A case is usually described by a pair tuple $\langle$ premise, value $\rangle$. Premise is the description of the case which contains its characteristics, while the value is the result of the reasoning based on the premise. A previously experience, which has been captured and learned, is referred to as a past case. Likewise, a new case is the description of a new problem to be solved. CBR is in fact a cyclic process of solving a problem, learning from this experience, solving a new problem as illustrated in Fig. 3. The premise part of a case referred in our situation-aware computing is a specific situation $S$ of a mobile user when using a search engine on his mobile device, while the value part of a case is the user profile $C$ to be used for the personalization of search results. Each case from our case base represents then a specific element from $U$, denoted : Case $=(S, C)$. Our CBR approach is involved across four steps process: (1) identifying the current case, (2) retrieving the most similar case, (3) reusing the case, (4) revising the proposed solution and/or retaining the case.

\subsection{Identifying the Current Case}

For a current query $q^{*}$ submitted to the search engine, a current case denoted Case $^{*}=\left(S^{*}, ?\right)$ is built. To represent the current situation $S^{*}$, sensory data

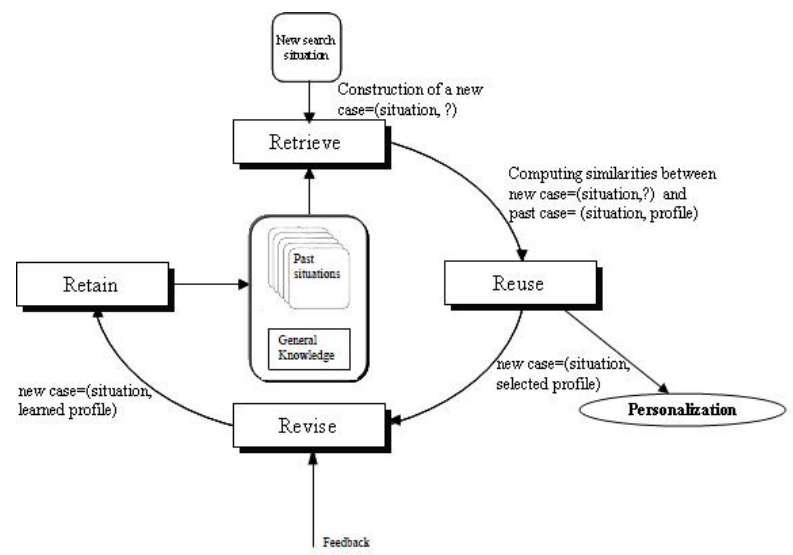

Fig. 3. CBR cycle 
related to the query $q^{*}$, are gathered from GPS sensor and system clock and then abstracted from the time and location ontologies, as outlined in section 3.2. We obtain then a semantic representation of $S^{*}$ :

$$
S^{*}=\left(X_{l}^{*}, X_{u}^{*}, X_{v}^{*}, X_{w}^{*}\right) \text {. }
$$

Case* $^{*}$ is then sent to the case base to complete its value part.

\subsection{Retrieve the Most Similar Case}

To determine the expected user profile in the current case Case*, the current situation $S^{*}$ is compared to the past ones. Let $P S=\left\{S^{1}, \ldots, S^{n}\right\}$ be the set of past situations, we select the situation $S^{\text {opt }}$ that verifies:

$$
S^{\text {opt }}=\underset{S^{i} \in P S}{\arg \max }\left(\sum_{j} \alpha_{j} \cdot \operatorname{sim}_{j}\left(X_{j}^{*}, X_{j}^{i}\right)\right)
$$

Where $X_{j}^{*}$ (resp. $X_{j}^{i}$ ) is the value of the $j^{\text {th }}$ feature of the situation vector $S^{*}$ (resp. $S^{i}$ ), $\operatorname{sim}_{j}$ is the similarity metric related to the $j^{\text {th }}$ feature of a situation vector and $\alpha_{j}$ its associated weight. These metrics are discussed below.

- Similarity for the location type dimension : For any situation $S$ the feature $X_{l}$ is given by a classification scheme of geographic places. The similarity between two location types $X_{l}^{*}$ and $X_{l}^{i}$ depends on how closely they are related in the taxonomy. Indeed, the similarity between museum and theater is greater than the similarity between museum and hospital. We use a similarity measure like in 23] which is defined by:

$$
\operatorname{sim}_{\text {location }}\left(X_{l}^{*}, X_{l}^{i}\right)=2 * \operatorname{depth}(l c s) /\left(\operatorname{depth}\left(X_{l}^{*}\right)+\operatorname{depth}\left(X_{l}^{i}\right)\right)
$$

where $l c s$ is the Least Common Subsumer of $X_{l}^{*}$ and $X_{l}^{i}$, and depth is the number of nodes on the path from a node to the root in the taxonomy.

- Similarity for the season dimension : For any situation $S$ the feature $X_{u}$ is an element of the predefined list \{autumn, winter, spring and summer $\}$. We assume situations within successive seasons to be more similar then situations within non successive ones. The similarity function between two seasons $X_{u}^{*}$ and $X_{u}^{i}$ is given by:

$$
\operatorname{sim}_{\text {season }}\left(X_{u}^{*}, X_{u}^{i}\right)= \begin{cases}1 & \text { if } X_{u}^{*}=X_{u}^{i} \\ 1 / 2 & \text { if } X_{u}^{*} \text { and } X_{u}^{i} \text { are successive seasons } \\ 0 & \text { otherwise }\end{cases}
$$

- Similarity for the day of the week dimension: For any situation $S$ the feature $X_{v}$ is an element of the predefined list $\{$ weekday, weekend, holiday $\}$. We assume situations within rest-days (holidays and weekends) to be more similar than situations within workdays. The similarity function between two seasons $X_{v}^{*}$ and $X_{v}^{i}$ is given by :

$$
\operatorname{sim}_{\text {week }}\left(X_{v}^{*}, X_{v}^{i}\right)= \begin{cases}1 & \text { if } X_{v}^{*}=X_{v}^{i} \\ 1 / 2 & \text { if } X_{v}^{*} \text { and } X_{v}^{i} \text { are rest }- \text { days } \\ 0 & \text { otherwise }\end{cases}
$$


- Similarity for the time of the day dimension : For any situation $S$ the feature $X_{w}$ is an element of the predefined list \{waking-time, morning, midday, afternoon, evening, night\}. We assume situations within work-times (morning and afternoon) to be more similar than situations within other day times. The similarity function between two seasons $X_{w}^{*}$ and $X_{w}^{i}$ is given by:

$$
\operatorname{sim}_{\text {day }}\left(X_{w}^{*}, X_{w}^{i}\right)= \begin{cases}1 & \text { if } X_{w}^{*}=X_{w}^{i} \\ 1 / 2 & \text { if } X_{w}^{*} \text { and } X_{w}^{i} \text { are work-times } \\ 0 & \text { otherwise }\end{cases}
$$

\subsection{Reuse the Case: Re-rank Search Results}

In order to insure a better precision of the research results, the personalization phase takes place only if the following condition is verified:

$$
\operatorname{sim}\left(S^{*}, S^{o p t}\right) \geq \beta \quad \text { where } \beta \text { is a threshold value. }
$$

The corresponding user's profile $C^{\text {opt }}$ is used to re-rank the search results returned by the search engine with respect to the current query $q^{*}$. The search results are re-ranked by combining for each retrieved document $d_{k}$, the original score returned by the system $\operatorname{score}_{o}\left(q^{*}, d_{k}\right)$ and a personalized score score $_{c}\left(d_{k}, C^{o p t}\right)$ obtaining a final score $_{f}\left(d_{k}\right)$ as follows:

$$
\text { score }_{f}\left(d_{k}\right)=\gamma * \operatorname{score}_{o}\left(q^{*}, d_{k}\right)+(1-\gamma) * \operatorname{score}_{c}\left(d_{k}, C^{o p t}\right)
$$

Where $\gamma$ ranges from 0 to 1 . Both personalized and original scores could be bounded by varying the values of $\gamma$. The personalized score $\operatorname{score}_{c}\left(d_{k}, C^{o p t}\right)$ is computed using the cosine similarity measure between the result $d_{k}$ and the top ranked concepts of the user profile $C^{\text {opt }}$ as follows:

$$
\operatorname{score}_{c}\left(d_{k}, C^{o p t}\right)=\sum_{c_{j} \in C^{o p t}} s w\left(c_{j}\right) * \cos \left(\overrightarrow{d_{k}}, \overrightarrow{c_{j}}\right)
$$

Where $s w\left(c_{j}\right)$ is the similarity weight of the concept $c_{j}$ in the user profile $C^{o p t}$.

\subsection{Revise the Proposed Solution and/or Retain the Case}

The case base is updated based on the user feedback which is used to learn the user profile $C^{*}$ for the search activity related to the current query $q^{*}$ (like described in section 3.3). Depending on the similarity value between the current situation $S^{*}$ and the most similar one $S^{o p t}$, two scenarios are plausible:

1. $\operatorname{sim}\left(S^{*}, S^{o p t}\right) \neq 1$ : a new case is added to the case base which is composed of the current situation $S^{*}$ with its learned profile $C^{*}$.

2. $\operatorname{sim}\left(S^{*}, S^{o p t}\right)=1$ : the case containing the situation $S^{o p t}$ is updated. Let $C^{\text {opt }}$ and $C^{*}$ be the user profiles for the search activities related to the same situation $S^{o p t}$. The updating method is based on the following principles: (1) enhance the weight of possible common concepts that can appear in 
two profiles related to the same $S^{o p t}$, (2) alter the weight of non-common concepts using a decay factor $\eta$. The new weight of a concept $c_{j}$ in the user profile $C^{o p t}$ is computed as follows:

$$
s w_{c^{o p t}}\left(c_{j}\right)=\left\{\begin{array}{l}
\eta * s w_{c^{o p t}}\left(c_{j}\right)+(1-\eta) * s w_{c^{*}}\left(c_{j}\right) \text { if } c_{j} \in C^{o p t} \\
\eta * s w_{c^{*}}\left(c_{j}\right) \text { otherwise }
\end{array}\right.
$$

where $s w_{c^{o p t}}\left(c_{j}\right)$ is the weight of concept $c_{j}$ in the profile $C^{o p t}$ and $s w_{c^{*}}\left(c_{j}\right)$ is the weight of concept $c_{j}$ in the profile $C^{*}$.

\section{Conclusion and Future Work}

This paper describes our approach for a situation-aware personalized search. It consists of three basic steps: (1) inferring semantic situations from low level location and time data, (2) learning and maintaining user interests based on his search history related to the identified situations, (3) selecting a profile to use for personalization given a new situation by exploiting a CBR technique. In future we plan to design an appropriate evaluation framework for mobile IR, and then undergo experiments in order to evaluate the impact of introducing the spatio-temporal user profiles in personalizing search results.

\section{References}

1. Kamvar, M., Baluja, S.: Deciphering trends in mobile search. Computer 40(8) (2007)

2. Sohn, T., Li, K.A., Griswold, W.G., Hollan, J.D.: A Diary Study of Mobile Information Needs. In: CHI 2008, Florence, Italy (April 5-10, 2008)

3. Bierig, R., Goker, A.: Time, location and interest: an empirical and user-centred study, vol. IIiX, pp. 79-87 (2006)

4. Goker, A., Myrhaug, H.I.: Evaluation of a mobile information system in context. Inf. Process. Manage. 44(1), 39-65 (2008)

5. Schofield, E., Kubin, G.: On Interfaces for Mobile Information Retrieval. In: Paternó, F. (ed.) Mobile HCI 2002. LNCS, vol. 2411, pp. 383-387. Springer, Heidelberg (2002)

6. Kamvar, M., Baluja, S.: The Role of Context in Query Input: Using contextual signals to complete queries on mobile devices. Mobile HCI, Human Computer Interaction with Mobile Devices and Services (2007)

7. August, K.G., Hansen, M.H., Shriver, E.: Mobile Web Searching. Bell Labs Technical Journal 6(2) (2002); Lucent Technologies Inc., published by Wiley Periodicals Inc.

8. Sweeney, S., Crestani, F.: Effective search results summary size and device screen size: Is there a relationship? Information Processing and Management 42(4) (2006)

9. Varma, V., Sriharsha, N., Pingali, P.: Personalized web search engine for mobile devices. In: Int'l Workshop on Intelligent Information Access, IIIA 2006 (2006)

10. Samaras, G., Panayiotou, C.: Personalized portals for the wireless user based on mobile agents. In: Proc. $2^{\text {nd }}$ Int'l workshop on Mobile Commerce, pp. 70-74 (2002) 
11. Yau, S.S., Liu, H., Huang, D., Yao, Y.: Situation-aware personalized information retrieval for mobile Internet. In: Proc. of the $27^{\text {th }}$ Annual International Computer Softwareand Applications Conference (COMPSAC 2003), pp. 638-645 (November 2003)

12. Panayiotou, C., Samaras, G.: Mobile User Personalization with Dynamic Profiles: Time and Activity. In: Meersman, R., Tari, Z., Herrero, P. (eds.) OTM 2006 Workshops. LNCS, vol. 4278, pp. 1295-1304. Springer, Heidelberg (2006)

13. Daoud, M., Tamine, L., Boughanem, M., Chebaro, B.: A Session Based Personalized Search Using An Ontological User Profile. In: ACM Symposium on Applied Computing (SAC 2009), pp. 1031-1035. ACM, New York (2009)

14. Anderson, C.R., Domingos, P., Weld, D.S.: Personalizing Web Sites for Mobile Users. In: Proc. 10th Int'l conf. on World Wide Web, Hong Kong, pp. 565-575 (2001)

15. Ala-Siuru, P., Rantakokko, T.: Understanding and recognizing usage situations using context data available in mobile phones. In: ubiPCMM 2006: 2nd Int'l Workshop on Personalized Context Modeling and Management for UbiComp Applications (2006)

16. Bila, N., Cao, J., Dinoff, R., Ho, T.K., Hull, R., Kumar, B., Santos, P.: Mobile User Profile Acquisition Through Network Observables and Explicit User Queries. In: proc. of the $9^{\text {th }}$ Int'l conference on Mobile Data Management, pp. 98-107 (2008)

17. Bellotti, V., Begole, B., Chi, E.H., Ducheneaut, N., Fang, J., Isaacs, E., King, T., Newman, M.W., Partridge, K., Price, B., Rasmussen, P., Roberts, M., Schiano, D.J., Walendowski, A.: Activity-Based Serendipitous Recommendations with the Magitti Mobile Leisure Guide. In: CHI 2008 Proceedings on the Move (2008)

18. Heckmann, D.: Ubiquitous User Modeling. Ph.D thesis, Saarland University (2005)

19. Park, T.: Toward a theory of user-based relevance: A call for a new paradigm of inquiry. J. Am. Soc. Inf. Sci. 45(3), 135-141 (1994)

20. Dobson, S.: Leveraging the subtleties of location. In: Proc. of Smart Objects and Ambient Intelligence, pp. 189-193 (2005)

21. Pan, F.: Representing complex temporal phenomena for the semantic web and natural language. Ph.D thesis, University of Southern California (December 2007)

22. Aamodt, A., Plaza, E.: Case-Based Reasoning: Foundational Issues, Methodological Variations, and System Approaches. AI Communications 7(1) (1994)

23. Wu, Z., Palmer, M.: Verb Semantics and Lexical Selection. In: Proceedings of the $32^{n d}$ Annual Meeting of the Association for Computational Linguistics (1994) 